\title{
FIXED SUBALGEBRA OF A COMMUTATIVE FROBENIUS ALGEBRA
}

\author{
GORO AZUMAYA
}

\begin{abstract}
Let $B$ be a finite-dimensional commutative algebra generated by a single element, and let $A=B \otimes B$. We prove that the fixed subalgebra of $A$ under the involution $b_{1} \otimes b_{2} \mapsto b_{2} \otimes b_{1}$ is Frobenius if and only if either the characteristic of $B$ is different from 2 or $B$ is separable.
\end{abstract}

1. Introduction. Let $A$ be a (finite-dimensional) Frobenius algebra over a field and $G$ a finite group of automorphisms of $A$. As is shown by $\mathrm{J}$. L. Pascaud and $\mathrm{J}$. Valette [3], it does not necessarily follow then that the fixed subalgebra $A^{G}$ under $G$ is Frobenius; indeed, they give an example of a local commutative Frobenius $A$ of any characteristic different from 2 and a group $G$ of order 2 such that $A^{G}$ is not Frobenius. (Note that for commutative algebras the concepts of "Frobenius" and "quasi-Frobenius" coincide.) In connection with this, N. Jacobson has raised the following question: Suppose $B$ is a commutative Frobenius algebra, or more particularly, an algebra generated by a single element, $A=B \otimes B$, and $G$ is the group of order 2 generated by the automorphism $\sigma: b_{1} \otimes b_{2} \mapsto b_{2} \otimes b_{1}$ of $A$. Is then $A^{G}$ Frobenius too? In the present paper the question is answered affirmatively in the case where $B$ is singly generated and the characteristic of $B$ is different from 2.

2. Lemmas. Let $B$ be a finite-dimensional commutative algebra over a field $K$, $A=B \otimes_{K} B$ and $G=\{1, \sigma\}$, where $\sigma$ is the automorphism as above. Suppose that $w_{1}, w_{2}, \ldots, w_{n}$ are a basis of $B$ over $K$. Then the $n^{2}$ elements $w_{i} \otimes w_{j}(1<i, j<n)$ form a basis of $A$. If we observe that $\left(w_{i} \otimes w_{j}\right)^{\circ}=w_{j} \otimes w_{i}$ for all $i, j$, we can easily derive the following

LemMA 1. Let $w_{1}, w_{2}, \ldots, w_{n}$ be a basis of $B$ over $K$. Then the $n$ elements $w_{i} \otimes w_{i}$ $(1<i<n)$ and $n(n-1) / 2$ elements $w_{i} \otimes w_{j}+w_{j} \otimes w_{i}(1<i<j<n)$ together form a basis of $A^{G}$ over $K$.

Let $L$ be an extension field of $K$. Then $B \otimes_{K} L$ is considered a commutative algebra over $L$, and we have $A \otimes_{K} L=\left(B \otimes_{K} L\right) \otimes_{L}\left(B \otimes_{K} L\right)$. The automorphism $\sigma$ of $A$ can also be regarded as an automorphism of $A \otimes_{K} L$ in the natural manner. Moreover, if $w_{1}, w_{2}, \ldots, w_{n}$ are a basis of $B$ over $K$ then it is also a basis

Received by the editors April 3, 1980.

AMS (MOS) subject classifications (1970). Primary 16A36, 16A72, 16A74.

Key words and phrases. Finite group of automorphisms acting on a ring, Frobenius algebra, characteristic different from 2. 
of $B \otimes_{K} L$ over $L$, and therefore by applying Lemma 1 to $B \otimes_{K} L$ and to this basis we know that the $n+n(n-1) / 2(=n(n+1) / 2)$ elements given in Lemma 1 together form a basis of $\left(A \otimes_{K} L\right)^{G}$ over $L$. Thus we have

Lemma 2. Let $L$ be an extension field of $K$. Then $\left(A \otimes_{K} L\right)^{G}=A^{G} \otimes_{K} L$.

Let $B=B_{1} \oplus B_{2} \oplus \cdots \oplus B_{r}$ be a decomposition of $B$ into a direct sum of orthogonal subalgebras. Put $A_{\alpha, \beta}=B_{\alpha} \otimes_{K} B_{\beta}$ for every $\alpha, \beta(=1,2, \ldots, r)$. Then $A_{\alpha, \beta}$ 's are orthogonal subalgebras of $A$ such that $A=\Sigma \oplus A_{\alpha, \beta}$. Each $A_{\alpha, \beta}$ clearly satisfies $A_{\alpha, \beta}^{o}=A_{\beta, \alpha}$, and in particular every $A_{\alpha, \alpha}$ is a $G$-invariant subalgebra. Let $(\alpha, \beta)$ be a pair such that $1<\alpha<\beta<r$, and put $\tilde{A}_{\alpha, \beta}=A_{\alpha, \beta} \oplus A_{\beta, \alpha}$. Then $\tilde{A}_{\alpha, \beta}$ is also a $G$-invariant subalgebra, and we have $\tilde{A}_{\alpha, \beta}^{G}=\left\{a+a^{\sigma} \mid a \in A_{\alpha, \beta}\right\}$, since $A_{\alpha, \beta}$ and $A_{\beta, \alpha}$ are orthogonal; thus we know that, by associating $a \in A_{\alpha, \beta}$ with $a+a^{\sigma}$, $\tilde{A}_{\alpha, \beta}^{G}$ is isomorphic to $A_{\alpha, \beta}$ as algebras. We have therefore the following

LEMMA 3. Let $B$ be a direct sum of orthogonal subalgebras $B_{\alpha}, 1<\alpha<r$. Let $A_{\alpha, \beta}=B_{\alpha} \otimes_{K} B_{\beta}$ for every $\alpha, \beta$, and let $\tilde{A}_{\alpha, \beta}=A_{\alpha, \beta} \oplus A_{\beta_{\alpha}}$ for every $\alpha, \beta$ such that $1<\alpha<\beta \leqslant r$. Then the $r(r+1) / 2$ algebras $A_{\alpha, \alpha}$ and $A_{\alpha, \beta}$ are orthogonal $G$-invariant subalgebras of $A=B \otimes_{K} B$, and $A$ is a direct sum of these subalgebras; moreover, every algebra $\tilde{A}_{\alpha, \beta}^{G}$ is isomorphic to $A_{\alpha, \beta}$.

Lemma 4. Let $B$ be a commutative Frobenius algebra, and let $A=B \otimes_{K} B$. Then $A^{G}$ is Frobenius if and only if $A_{\alpha, \alpha}^{G}$ is Frobenius for every $\alpha$.

Proof. From Lemma 3 it follows that $A^{G}$ is the direct sum of orthogonal subalgebras $A_{\alpha, \alpha}^{G}$ and $\tilde{A}_{\alpha, \beta}^{G}$, where the latter algebras are isomorphic to $A_{\alpha, \beta}$. Therefore $A^{G}$ is Frobenius if and only if all $A_{\alpha, \alpha}^{G}$ and $A_{\alpha, \beta}$ are Frobenius. Since however $B$ is a Frobenius algebra, each direct summand subalgebra $B_{\alpha}$ and hence $A_{\alpha, \beta}=B_{\alpha} \otimes_{K} B_{\beta}$ are Frobenius algebras too. Therefore, in order that $A^{G}$ be Frobenius it is (necessary and) sufficient that $A_{\alpha, \alpha}^{G}$ be Frobenius for all $\alpha$.

\section{Main results.}

Proposition 5. Let $B$ be an algebra generated by a nonzero nilpotent element over a field $K$. Then $A^{G}$ is a Frobenius algebra if and only if the characteristic of $K$ is different from 2.

Proof. Let $B=K[c]$ with a nilpotent element $c \neq 0$ of $B$. Let $n$ be the least positive integer such that $c^{n}=0$. Then $n>2$, and the $n$ elements $1, c, c^{2}, \ldots, c^{n-1}$ form a basis of $B$ over $K$. Therefore, by Lemma 1, the $n$ elements $c^{i} \otimes c^{i}(0 \leqslant i \leqslant n-1)$ and $n(n-1) / 2$ elements $c^{i} \otimes c^{j}+c^{j} \otimes c^{i}(0<$ $i<j \leqslant n-1$ ) together form a basis of $A^{G}$ over $K$. If we remove $1 \otimes 1$ (the unit element of $A^{G}$ ) out of the basis then we have a subset $W$. Let $N$ be the $K$-subspace of $A^{G}$ generated by $W$. Then since every element of $W$ is nilpotent, $N$ is contained in the radical of $A^{G}$. It is however clear that $A^{G}=N \oplus K(1 \otimes 1)$, and this, together with the fact that the radical is a proper ideal of $A^{G}$, implies that $N$ coincides with the radical and the factor algebra $A^{G} / N$ is isomorphic to $K$. Thus, $A^{G}$ is a local ring and has an up-to-isomorphism unique simple module, which is one-dimensional over $K$. Moreover, $N$ is the ideal of $A^{G}$ generated by $c \otimes c$ and 
$1 \otimes c+c \otimes 1$, because $c^{i} \otimes c^{i}=(c \otimes c)^{i}$ for $i>0$,

$$
\begin{aligned}
1 \otimes c^{2}+c^{2} \otimes 1 & =(1 \otimes c+c \otimes 1)^{2}-2(c \otimes c) \\
1 \otimes c^{i}+c^{i} \otimes 1= & (1 \otimes c+c \otimes 1)\left(1 \otimes c^{i-1}+c^{i-1} \otimes 1\right) \\
& -(c \otimes c)\left(1 \otimes c^{i-2}+c^{i-2} \otimes 1\right) \text { for } i>2
\end{aligned}
$$

and

$$
c^{i} \otimes c^{j}+c^{j} \otimes c^{i}=(c \otimes c)^{i}\left(1 \otimes c^{j-i}+c^{j-i} \otimes 1\right) \text { for } j>i>0 .
$$

(More precisely, by using these equalities, it is possible to show without difficulty that every element of $W$ is expressed as a polynomial of $c \otimes c$ and $1 \otimes c+c \otimes 1$ with integral coefficients and without constant term.)

Let now $M$ be the socle of $A^{G}$, i.e., the sum of all simple ideals of $A^{G}$. As is well known, $M$ is then the annihilator of the radical $N$ in $A^{G}$ and therefore is the annihilator of the generators $c \otimes c$ and $1 \otimes c+c \otimes 1$ of $N$ in $A^{G}$. Let $M^{\prime}$ be the annihilator of $c \otimes c$ in $A^{G}$. If we observe that

$$
(c \otimes c)\left(c^{i} \otimes c^{i}\right)=c^{i+1} \otimes c^{i+1}
$$

is a member of $W$ or $=0$ according as $0<i<n-2$ or $j=n-1$ and also

$$
(c \otimes c)\left(c^{i} \otimes c^{j}+c^{j} \otimes c^{i}\right)=c^{i+1} \otimes c^{j+1}+c^{j+1} \otimes c^{i+1}
$$

is a member of $W$ or $=0$ according as $0<i<j \leqslant n-2$ or $j=n-1$, then we know that $M^{\prime}$ is the $K$-subspace of $A^{G}$ generated by $c^{n-1} \otimes c^{n-1}, 1 \otimes c^{n-1}+$ $c^{n-1} \otimes 1, c \otimes c^{n-1}+c^{n-1} \otimes c, \ldots, c^{n-2} \otimes c^{n-1}+c^{n-1} \otimes c^{n-2}$. Next multiply these $n$ elements by $1 \otimes c+c \otimes 1$. Then we have

$$
\begin{gathered}
(1 \otimes c+c \otimes 1)\left(c^{n-1} \otimes c^{n-1}\right)=0, \\
(1 \otimes c+c \otimes 1)\left(1 \otimes c^{n-1}+c^{n-1} \otimes 1\right)=c \otimes c^{n-1}+c^{n-1} \otimes c, \ldots, \\
(1 \otimes c+c \otimes 1)\left(c^{n-3} \otimes c^{n-1}+c^{n-1} \otimes c^{n-3}\right)=c^{n-2} \otimes c^{n-1}+c^{n-1} \otimes c^{n-2}, \\
(1 \otimes c+c \otimes 1)\left(c^{n-2} \otimes c^{n-1}+c^{n-1} \otimes c^{n-2}\right)=2\left(c^{n-1} \otimes c^{n-1}\right),
\end{gathered}
$$

where the last element is 0 if and only if the characteristic of $K$ is 2. This means that the annihilator of $1 \otimes c+c \otimes 1$ in $M^{\prime}$, i.e., the annihilator $M$ of $N$ in $A^{G}$ is $K \cdot\left(c^{n-1} \otimes c^{n-1}\right)$ or $K \cdot\left(c^{n-1} \otimes c^{n-1}\right) \oplus K \cdot\left(c^{n-2} \otimes c^{n-1}+c^{n-1} \otimes c^{n-2}\right)$ according as the characteristic of $K$ is different from 2 or equal to 2. Now, since $A^{G}$ is a local ring, $A^{G}$ is a Frobenius algebra if and only if $A^{G}$ has a unique simple ideal, or equivalently, its socle $M$ is simple. But since $A^{G} / N$ is isomorphic to $K$, this is equivalent to the condition that $M$ is one-dimensional over $K$. Thus we have that $A^{G}$ is Frobenius if and only if $M=K \cdot\left(c^{n-1} \otimes c^{n-1}\right)$, i.e., the characteristic of $K$ is different from 2 .

Corollary 6. Let $B=K[a]$, and let the minimal polynomial $f(t)$ of a over $K$ be a power of a linear polynomial in $K$. Then $A^{G}$ is a Frobenius algebra if and only if the characteristic of $K$ is different from 2 or $f(t)$ is linear.

Proof. Let $f(t)=(t-\lambda)^{n}, \lambda \in K$. If we put $c=a-\lambda$ then $B=K[c]$ and $n$ is the least positive integer such that $c^{n}=0$. Thus, if $n>1$ then $c \neq 0$ and by Proposition $5, A^{G}$ is Frobenius if and only if the characteristic of $K$ is different 
from 2, while if $n=1$ then $c=0$ and clearly $B=K=A=A^{G}$ and the $K$-algebra $K$ is trivially a Frobenius algebra.

THeOREM 7. Let $B=K[a]$ and let $f(t)$ be the minimal polynomial of a over $K$. Let $A=B \otimes_{K} B$ and let $G=\{1, \sigma\}$, where $\sigma$ is the automorphism of $A$ defined by $\left(b_{1} \otimes b_{2}\right)^{\circ}=b_{2} \otimes b_{1}$ for $b_{1}, b_{2} \in B$. Then $A^{G}$ is a Frobenius algebra over $K$ if and only if the characteristic of $K$ is different from 2 or $f(t)$ is separable (i.e., $f(t)$ has no multiple root in a splitting field).

Proof. (i) Assume first that $K$ is algebraically closed. Then $f(t)$ is decomposed as

$$
f(t)=\left(t-\lambda_{1}\right)^{n_{1}}\left(t-\lambda_{2}\right)^{n_{2}} \cdots\left(t-\lambda_{r}\right)^{n_{r}},
$$

where $\lambda_{1}, \lambda_{2}, \ldots, \lambda_{r}$ are distinct roots of $f(t)$ in $K$. As is well known, this decomposition yields a direct decomposition $B=B_{1} \oplus B_{2} \oplus \cdots \oplus B_{r}$ into orthogonal subalgebras such that if $a_{\alpha}$ is the $B_{\alpha}$-component of $a$ then $B_{\alpha}=K\left[a_{\alpha}\right]$ and $\left(t-\lambda_{\alpha}\right)^{n_{\alpha}}$ is the minimal polynomial of $a_{\alpha}$ over $K$ for every $\alpha$. Now, as a homomorphic image of the polynomial ring $K[t], B$ is a principal ideal ring and therefore a Frobenius algebra (cf. Nakayama [1, Theorem 16]). From this follows by Lemma 4 that $A^{G}$ is Frobenius if and only if $A_{\alpha, \alpha}^{G}$, where $A_{\alpha, \alpha}=B_{\alpha} \otimes_{K} B_{\alpha}$, is Frobenius for every $\alpha$. On the other hand, according to Corollary 6, each $A_{\alpha, \alpha}^{G}$ is Frobenius if and only if the characteristic of $K$ is different from 2 or $n_{\alpha}=1$. Thus we have that $A^{G}$ is a Frobenius algebra if and only if either the characteristic of $K$ is different from 2 or $n_{1}=n_{2}=\cdots=n_{r}=1$.

(ii) Suppose that $K$ is not necessarily algebraically closed. Let $L$ be the algebraic closure of $K$. Then $\left(A \otimes_{K} L\right)^{G}=A^{G} \otimes_{K} L$ by Lemma 2. Therefore it follows by Nakayama [1, Theorem 14] or Nakayama and Nesbitt [2, Theorem 5] that $A^{G}$ is Frobenius over $K$ if and only if $\left(A \otimes_{K} L\right)^{G}$ is Frobenius over $L$. But if we observe that $A \otimes_{K} L=\left(B \otimes_{K} L\right) \otimes_{L}\left(B \otimes_{K} L\right), B \otimes_{K} L=L[a]$ and the minimal polynomial of $a$ over $L$ is the same as $f(t)$, we can conclude by the above case (i) that the latter condition is equivalent to the condition that the characteristic of $L$ whence of $K$ is different from 2 and $f(t)$ has no multiple root in $L$. This completes the proof of our Theorem.

\section{REFERENCES}

1. T. Nakayama, On Frobeniusean algebras. II, Ann. of Math. 42 (1941), 1-21.

2. T. Nakayama and C. J. Nesbitt, Note on symmetric algebras, Ann. of Math. 39 (1938), 659-668.

3. J. L. Pascaud and J. Valette, Group actions on Q-F-rings, Proc. Amer. Math. Soc. 76 (1979), $43-44$.

Department of Mathematics, Indiana University, Bloommgton, Indiana 47401 